\title{
La religión de los antiguos persas en las filosofías de la historia*
}

\author{
( Borja Antela-Bernárdez \\ Universitat Autònoma de Barcelona
}

Fecha de recepción: 28 de julio de 2018. Fecha de aceptación: 15 de agosto de 2018.

\begin{abstract}
Resumen
Los antiguos persas habitualmente han ocupado un espacio marginal, de alteridad, en tanto que opuestos al modelo histórico representado por Grecia, en las teorías sobre la historia y el progreso. A través de una revisión de la opinión reflejada sobre los antiguos persas en obras sobre las filosofías de la historia, se pretende poner de manifiesto que uno de los valores propios de los Aqueménidas, como es el de la religión, ha sido valorado positivamente. Las razones de esta paradoja entre persas como negativos pero positiva su religión puede ayudarnos a entender ciertas percepciones y apriorismos en la relación de estas ideas con la historiografía.
\end{abstract}

\begin{abstract}
The ancient Persians have usually had a marginal space, as symbols of alterity, as a consequence of their traditional opposition with the historical model represented by Greece, in the theories of history and progress. Through a review of the opinion about the ancient Persians reflected in works on the philosophies of history, the aim of this paper is to show that one of the elements of the ancient Achaemenids, as it is their religion, has been perceived as positive. The reasons of this paradox between Persians as negatives but their religion as positive can help us to understand some perceptions and aprioristic views in the relationship of this ideas with the historiography.
\end{abstract}

\footnotetext{
* Investigación desarrollada dentro del Grup de Recerca Història del Conflicte a l'Antiguitat (2017SGR234), reconocido y financiado por la Generalitat de Catalunya. Este trabajo está dedicado a mi maestro, X. C. Bermejo Barrera, y a Pierre Briant, por la inspiración de ambos.
}

Palabras clave

Persas

Aqueménidas Religión

Zoroastrismo monoteísmo filosofías de la historia

Keywords

Persians Achaemenids Religion Zoroastrism Monotheism Philosophies of History 
1. Kant, $A A$ XV: 878: “Unsere (alte) Geschichte der Menschen geht mit Zuverläßigkeit nur auf die Race der Weissen. Egypter. Perser. Thracier. Griechen. Celten. Scythen. (nicht Indier, Neger)"; cf. $\mathrm{M}^{\mathrm{a}}$ J. Vázquez Lobeiras (2008: 218).

2. Bossuet (1996: 379).
A la luz de la historiografía, la religión de los antiguos persas ha sido uno de los elementos valiosos en el devenir histórico de la antigua Persia, así como el lugar del Imperio Aqueménida en la sucesión de sistemas políticos y la evolución de la humanidad planteados por las filosofías de la historia, y como resultado de éstas, también por las interpretaciones que los historiadores de las diversas épocas han articulado en relación con los persas del mundo antiguo. Es nuestra intención señalar y analizar aquí el valor que se ha asociado a la religión de los Aqueménidas y valorar este parámetro en relación con el papel cumplido por estos en las percepciones historiográficas. Partiremos para ello de un contexto muy concreto como es el de las filosofías aplicadas a la comprensión del pasado y del devenir de la Humanidad, en la búsqueda de un sentido para la Historia. Es a través de estas "Filosofías de la Historia" que rastrearemos el lugar asignado a los persas en el decurso de la historia universal, y trataremos de advertir el valor de la religión persa como elemento de peso en esta asignación.

En primer lugar, vale la pena recordar que la construcción de las Filosofías de la Historia de los s. XVII a XIX concibe la Historia como una sucesión de pueblos (que no incluye a todos los pueblos), donde dicha sucesión está dotada de sentido y el objetivo de la misma es la evolución o la perfección de la humanidad. En esta línea evolutiva de los pueblos, los persas tienen un lugar reservado. Escribe Kant:

Nuestra historia de la humanidad concierne con fiabilidad únicamente a la raza de los blancos: egipcios, persas, tracios, griegos, escitas (no a los indios y a los negros). ${ }^{1}$

En ello tiene mucho que ver el papel de los persas como unificadores de Oriente, aunque esta unificación reciba muchos matices y no pueda compararse con la que Roma llevará a cabo en Occidente, como expone claramente Bossuet:

Leur grand empire fut toujours régi avec qualque confusion. Ils ne se surent jamais trouver ce bel art, depuis si bien pratiqué par les Romains, d'unir toutes les parties d'un grand Etat, et d'en faire un tout parfait. Aussi n'étaient-ils presque jamais sans revoltes considerables. ${ }^{2}$

En cuanto a la religión persa, Bossuet señala:

Le respect qu'on inspirait aux Perses, dès leur enfance, pour l'autorité royale, allait jusqu'à l'excès, puisqu'ils y mêlaient de l'adoration, et paraissaient plutôt des esclaves que des sujets soumis par raison à un empire légitime: c'était l'esprit des Orientaux; et peut-être que le natural vif et violent de ces peuples demandait un gouvernement plus ferme et plus absolu.

Esta afirmación señala que el elemento básico en Bossuet de la religión persa es el de garantizar el despotismo oriental de los reyes aqueménidas sobre unos pueblos que, como orientales, son por naturaleza violentos. Si bien a primera vista ello no parece tener demasiado valor a la hora de definir el carácter histórico de los persas, lo cierto es que estamos ante un ejemplo magnífico de lo que Edward Said ha descrito en sus trabajos sobre Orientalismo. Asimismo, Heleen Sancissi-Weerdenburg ha estudiado la influencia directa que la transmisión de la Ciropedia de 
Jenofonte ha tenido en la construcción europea de la imagen de Persia, componiendo así los persas uno de los más destacados ejemplos de alteridad oriental. ${ }^{3}$ Es por ello que no parece existir una gran distancia entre las percepciones y conocimientos de Bossuet sobre los persas en su Discurso sobre la historia universal y el Fifth Oriental Monarchy: Persia de Henry Rawlison, pese a que el primero fue publicado en 1681 y el segundo en 1871, más de dos siglos después.

La cuestión religiosa de los persas tiene un peso mayor en la obra de Voltaire, quien en su Essai sur les moeurs et l'esprit des nations, publicado en 1756, escribe:

Je remarquerai que les Parsis, ou Perses, prétendaient avoir eu parmi eux, il y avait six mille ans, un ancien Zerdust, un prophète, qui leur avait appris à être justes et à révérer le soleil, comme les anciens Chaldéens avaient révéré les étoiles en les observant.

Je me garderai bien d'affirmer que ces Perses et ces Chaldéens fussent si justes, et de déterminer précisément en quel temps vint leur second Zerdust, qui rectifia le culte du soleil, et leur apprit à n'adorer que le Dieu auteur du soleil et des étoiles. II écrivit ou commenta, dit-on, le libre du Zend, que les Parsis, dispersés aujourd'hui dans l'Asie, révèrent comme leur Bible. (...)

J'y vois que ces Parsis croyaient depuis longtemps un dieu, un diable, une résurrection, un paradis, un enfer. Ils sont les premiers, sans contredit, qui ont établi ces idées; c'est le système le plus Antique, et qui ne fut adopté par les autres nations qu'après, bien des siècles, puis les pharisiens, chez les Juifs, ne soutinrent hautement l'immortalité de l'âme, et le dôme des peines et des récompenses après la mort, que vers le temps des Asmonéens.

Voilà peut-être ce qu'il y a de plus important dans l'ancienne histoire du monde: voilà une religion utile, établie sur le dogme de l'immortalité de l'âme et sur la connaissance de l'Être créateur. Ne cessons point de remarquer par combien de degrés il fallut que l'esprit humain passât pour concevoir un tel système. Remarquons encore que le baptême (l'immersion dans l'eau pour purifier l'âme par le corps) est un des préceptes du Zend (porte 251). La source de tous les rites est venue peut-être des Persans et des Chaldéens, jusqu'aux extrémités de la terre. ${ }^{4}$

De forma evidente, la imagen positiva de los persas en Voltaire es resultado de su tradición religiosa, y ésta es considerada en tanto que cercana a la del propio Voltaire. Es decir, la religión persa es considerada en términos positivos por sus similitudes con la de occidente de tiempos de Voltaire. Ésta será la tendencia fundamental en la filosofía de la historia de las religiones, en especial en aquellas focalizadas en lo que Bermejo Barrera denomina las religiones del Espíritu, ${ }^{5}$ que tienen por otra parte un referente fundamental, e incluso fundacional (si tenemos en cuenta su influencia directa en Bossuet) en La ciudad de Dios de San Agustín, quien describe a los persas por medio de su religión:

Pues bien, aquél único Dios verdadero que ni en juicio ni en ayuda desampara al linaje humano, cuando quiso y cuanto quiso, dio la hegemonía a los romanos; Él fue quien la dio a los asirios y aun a los persas, de cuyos monumentos literarios consta que solamente adoraban a dos dioses, uno bueno y otro malo. ${ }^{6}$
3. Sancisi-Weenderburg (1987: 117): "The concept of the Orient is the end result of a long process of ethnocentric thinking in which one's own culture is regarded as the norm and everything outside it as deviating from or complementary to it. The Orient is for Europe 'one of its deepest and most recurring images of the Other'”.
4. Voltaire (1962, I: 40-41).

5. Bermejo Barrera (2009: 636). 
7. Herder (1959: 369-371). La negrita es mía.

8. Cf. Blok (1994).

9. Creuzer (1825, I: 319-330).
Volviendo a la filosofía de la historia, lo expuesto por Voltaire a mediados del s. XVIII mantiene su vigencia en la obra de Johan Gottfried Herder, quien entre 1784 y 1791 publicó su Ideas para una filosofía de la historia de la Humanidad. En Herder se mezclan las dos visiones anteriores que hemos visto, la política de Bossuet, y la religiosa, de Voltaire:

Su espíritu conquistador les había fijado un fin tan alto que persistieron en ampliar los límites del Imperio cuando esto ya no era posible. De esta manera acabaron por devastar y chocar con todos sus vecinos hasta que, por fin, el pundonor de un enemigo ofendido les deparó su triste final. Apenas dos siglos duró el Imperio Persa y uno se admira de que haya durado tanto, porque sus raíces eran tan débiles y sus ramas, en cambio, tan grandes, que forzosamente tuvo que derrumbarse el árbol.

Si alguna vez el sentido humanitario se hace lugar entre los hombres, lo primero que habrá que aprender de la historia de los persas es renunciar a la locura de un desenfrenado espíritu conquistador, que en pocas generaciones acaba por perderse a sí mismo. (...) Un Imperio juntado a punta de lanza, que abarca cien pueblos y ciento veinte provincias, no es un Estado sino un monstruo.

Tal fue la monarquía persa desde un principio.

(...) En consecuencia, este reino no ha ejercido influjo benéfico sobre otra nación alguna porque en vez de edificar destruía. (...) Hasta su historia se ha convertido en fábula, una fábula que los orientales y griegos prefieren no mencionar. También los antiguos idiomas persas han muerto (...). Lo único que nos habría quedado de los monumentos espirituales de los persas serían los libros de Zoroastro si su autenticidad estuviera demostrada. (...) Los antiguos persas, como todos los pueblos salvajes y particularmente los montañeses, veneraban los elementos vivos del mundo. Mas como este pueblo no permaneció en su incultura sino que ascendió por sus victorias hasta la más alta cumbre de la opulencia, la mentalidad asiática pedía ya un sistema mejor ideado o un ceremonial religioso; lo percibió finalmente de manos de su Zoroastro o Zerduscht, al que prestaba su apoyo el rey Darío Histaspes. El sistema se basa evidentemente en la Constitución persa; así como había siete príncipes alrededor del trono a manera de ministros, así hay aquí delante de Dios siete espíritus que ejecutan sus órdenes en todos los mundos. Ormuzd, el ser bueno, príncipe de la luz tiene que luchar incesantemente con Arimán, príncipe de las tinieblas, y en esta batalla le ayudan todos los buenos. ${ }^{7}$

También F. Creuzer, quien a principios del s. XIX publicó un influyente estudio sobre historia de las religiones, mantuvo una visión parecida a la que hemos ido percibiendo, que tuvo además un gran impacto en los historiadores de su tiempo, especialmente entre los alemanes, mediante una disputada diferencia interpretativa con K. O. Müller en la concepción científica de la nueva ciencia de las religiones que estaba naciendo en el s. XIX ${ }^{8}$. Así, Creuzer redunda en esa imagen de la religión persa como dual, como lucha entre luz y oscuridad, que simbolizan a su vez bien y mal. ${ }^{9}$

La percepción positiva de los persas en Herder tiene su continuación, y su consolidación en los sistemas que argumentarían la epistemología de la disciplina 
histórica, en la obra de Georg Friedrich Wilhelm Hegel. En sus Lecciones sobre la filosofía de la historia universal, Hegel reafirma el lugar privilegiado de los persas en la concepción de la historia como progreso de la humanidad.

Con el imperio persa comienza la franca conexión con la historia universal. (...) Los persas son el primer pueblo histórico; Persia es el primer imperio que ha sucumbido. (...) En Persia es donde el hombre empieza ya a separarse de la naturaleza. (...) Aquí en Persia, surge por primera vez la luz que brilla e ilumina otras cosas. La luz de Zoroastro es la primera que pertenece al mundo de la conciencia, al espíritu como referencia a otra cosa. También aquí existe la unidad de lo espiritual y lo natural; el mundo finito está comprendido en lo uno y lo natural en la luz. Pero esta unidad se cierne sobre la diversidad natural y, de esta suerte, surge la abstracción de la divinidad, junto a la cual todo lo demás solo tiene valor por cuanto es un rayo de la divinidad. Con esto queda establecida la divinidad de los principios. El bien, lo moral, consiste en que el fundamento de la acción sea lo universal; y todo cuanto se puede subsumir bajo el bien constituye este reino único de la luz. Encontramos en Persia la antítesis de la luz y las tinieblas, o del bien y del mal, de la divinidad y de lo finito. (...) El principio de la evolución se inicia con la historia de Persia; por eso esta historia constituye el verdadero comienzo de la historia universal, pues el interés universal del espíritu en la historia estriba en llegar al infinito "ser en sí" de la subjetividad, en llegar a la conciliación por medio de la absoluta antítesis. ${ }^{10}$

Más allá de la complejísima conceptualización teórica desplegada por Hegel, para nuestros propósitos parece evidente que el elemento fundamental del papel de Persia en la historia de la humanidad, para Hegel, proviene en realidad de este fenómeno religioso que él define como la oposición de la luz y las tinieblas, en clara referencia a lo que él considera la síntesis de la religión inaugurada por Zoroastro, esta religión de la $l u z^{11}$ que habíamos advertido ya en ejemplos anteriores.

Esta identificación de la religión aqueménida como cercana a la propia se mantiene, pese a los importantes cambios que vive el mundo durante el primer cuarto del siglo XX, en obras a priori más alejadas de esta filosofía de las religiones, aunque herederas directas de la filosofía de la historia, como son las de Spengler y Toynbee.

Sorprende profundamente el enorme espacio que los persas ocupan en la obra de Spengler La Decadencia de Occidente, tan influyente en diversos círculos intelectuales, incluso entre muchos historiadores. ${ }^{12}$ Más allá de las múltiples consideraciones que en esta obra aparecen sobre los persas, la lectura de proximidad entre el cristianismo occidental y la religión persa se mantiene de forma evidente, siendo los persas presentados como auténticos antecedentes:

The Persians indeed comprised the future as well in the sweep of the gesture, and predated the last Judgement and the coming of the Messiah. ${ }^{13}$

En un tono similar se expresa, pese a las diferencias que opusieron a ambos autores, Arnold Toynbee en su A Study of History:
10. Hegel (1994: 323-324).

11. Hegel (1994: 327).

12. Un buen ejemplo es el de Franz Altheim. Cf. AntelaBernárdez (en prensa).

13. Spengler (1926: 27-28). 
It looks as if the apparatus of political power which is wielded by the dominant minority so successfully for the purpose of controlling its subject population in the secular side of their life becomes a positive obstacle to the imposition of an official philosophical or religious doctrine. By contrast, the politically impotent internal proletariat can exploit most effectively the opportunity offered by the pacific atmosphere of a universal state for propagating, from below upwards, a higher religion and eventually establishing a universal church.

The Neo-Babylonian Empire and its successive avatars, the Achaemenian Empire and the Seleucid Monarchy, were used to this effect by Judaism and by its sisterreligion Zoroastrism. ${ }^{14}$

De este modo, hemos podido advertir cómo una percepción específica de la realidad histórica de los antiguos persas, centrada en su religión, se convierte en la razón del lugar que éstos ocuparon en la historia de la humanidad. Asimismo, hemos podido advertir también que esta percepción positiva de la religión persa se debe a una identificación de la misma como similar, preliminar o, sobre todo, proximal a la conciencia religiosa de aquellos autores que han desarrollado las corrientes básicas de la filosofía de la historia y la historia de las teorías de las religiones del espíritu, que ya hemos explicado. No obstante, esta observación contrasta con otras tres circunstancias.

La primera de ellas sería la pésima imagen que sobre los persas ha prevalecido a lo largo del tiempo, como fruto de la tradición textual griega, de la que el mundo europeo y sus descendientes han sido claros herederos. Existe toda una serie de trabajos recientes en los que autores, como Pierre Briant, ${ }^{15}$ han puesto de manifiesto estas construcciones historiográficas sobre la visión peyorativa sobre el imperio aqueménida a lo largo de los siglos desde el s. XVI, como mínimo. ${ }^{16}$

16. En un trabajo anterior, he reflexionado sobre esta imagen negativa, en relación con la percepción de los persas como esclavos: Antela-Bernárdez y Zaragozà (en prensa).

17. Sobre la formación de la actual aquemenología, véase Imanpour (2013; 2015).

18. Un ejemplo magnifico, por reciente y por el supuesto prestigio de su autor, es el del libro de Cartledge (2004), quien dedica un par de páginas (de la 69 a la 73 , sin contar la 72, que es un mapa) a explicar el imperio persa para que el lector pueda entender contra quién se enfrentaron los griegos, aunque para ello recurre a la manida tradición de historia política, con un breve y muy "herodoteo" relato sobre los reyes persas, pero sin precisar en modo alguno detalles que ayuden a hacerse una idea al lector sobre la realidad histórica que suponía la Persia aqueménida.
La segunda circunstancia tiene que ver con la impronta que estas construcciones teóricas de las filosofías de la historia pueden haber tenido en los historiadores e investigadores sobre la Antigüedad. En este sentido, vale la pena mencionar que hasta principios de la década de 1980 los estudios sobre la historia aqueménida no eran frecuentes entre los historiadores de la Antigüedad, ${ }^{17}$ y ello implicaba que muy a menudo las historias de Grecia sólo mencionasen a los persas en relación con los episodios de enfrentamiento con la Hélade, y para ello realizasen algún excurso breve o párrafos explicativos sobre el mundo aqueménida como introducción a una explicación que siempre trataba las cuestiones entre griegos y persas del lado helénico. ${ }^{18}$ Así, los persas, encarnación de la barbarie, eran descritos sólo por medio de pequeños apuntes que, en el fondo, tenían poco interés en comprender su realidad histórica, prestando más atención a la percepción griega que a la posibilidad de conocer el mundo persa históricamente por sí mismo y no sencillamente como antagonista de los griegos.

Quizás esta falta de interés, o este interés por desconocer, en realidad, a los persas en beneficio de los griegos explica que la percepción de los persas como zoroastristas monoteístas pueda haberse perpetuado a lo largo de tanto tiempo. Especialmente si tenemos en cuenta que dista mucho de ser correcta. No es 
este el lugar para analizar en detalle los elementos de la religión persa como tal, pero podemos citar la brevísima explicación de Maria Brosius sobre este tema como ejemplo:

The Persians regarded the natural elements, earth and sky, water and fire, as well as rivers and mountains, as sacred, a belief shared with the Iranian peoples like the Medes, as well as with the Elamites.

\section{(...)The early Persians worshipped many gods.}

(...)Though Ahuramazda was the principal god of the Achaemenids, he was not the only god. Inscriptions of Darius also include references to 'the other gods', though these are not specifically mentioned. The reference is too ambiguous to determine whether these include only other Iranian gods or the gods of the peoples of the empire. We do know, however, that in Persepolis itself other gods were worshipped. In addition to the worship of mountains, rivers and lakes, we find references to the cult of Elamite gods like Humban, and Babylonian gods like Adad.

(...) It seems that at a later stage, probably during the reign of Artaxerxes II, two further Iranian gods were elevated to the royal religious cult alongside Ahuramazda, Mithra, the sun-god and god of treaties, and Anahita, the goddess of water and fertility.

(...)Two important questions surround the cult of Ahuramazda: first, were the Achaemenids, if not Cyrus II and Cambyses II, already followers of Zoroastrianism, and second, who were the followers of the royal cult? Zoroastrianism, the religion preached by the prophet Zoroaster, is a religion characterised by the dualism between Good and Evil, Truth and Lie, presented in the good god, Ahuramazda, and Ahriman, the evil god. Men's lives are a struggle between these two powers, but they hope to achieve redemption and enter Paradise after death. The sayings of the prophet were collected in the Avesta, the Holy Scriptures, a collection of different texts used in Zoroastrian ritual.

(...)The Persians, like the Elamites, believed in many divinities, represented in the natural elements and celestial constellations. These were combined with a pantheon of Iranian deities, whose cults had been introduced to Persia over the long period of migration from the east and northeast. Ahuramazda, like Mithra and Anahita, was one such deity, whose cult probably originated in the Eastern part of the Persian empire, but only received an exalted status under the Achaemenid kings beginning with Darius I.

This religion is probably best described as Mazdaism, the belief in Mazda, the Lord. ${ }^{19}$

Parece que existe, pues, una confusión entre lo que los filósofos que hemos visto consideran religión persa o zoroastrismo y lo que fue en realidad la religión de los aqueménidas. ${ }^{20} \mathrm{M}$. Brosius lo explica también:

The Persian king was not a god-king, but he ruled with the support of the god Ahuramazda, the 'Wise Lord'. Ahuramazda was an Iranian deity whose cult was elevated to a royal religious cult at the time of Darius I. As the god's representative

20. Sobre la religión de los antiguos persas puede revisarse el libro de Malandra (1983). 
21. Brosius (2006: 32-33).

22. Un análisis de la imagen del zoroastrismo en las fuentes grecorromanas ha sido llevado a cabo por de Jong (1997). Para un análisis más reciente sobre esta cuestión y sus problemas, véase Kreyenbroek (2005).

23. Gordon Childe (1926: 209); cf. Samiei (2014: 209).

24. Briant (2016).

25. Véase Antela-Bernárdez (en prensa). Para el caso de Persia y el nazismo, véase asimismo un ejemplo en Pringle (2006: 183).

26. Motadel (2013). Asimismo, para una definición e historia del arianismo, véase Zia-Ebrahimi (2011). Igualmente, me ha gustado el trabajo de Zia-Ebrahimi sobre la relación entre memoria e historiografía en Irán: Zia-Ebrahimi (2016).

27. Morris (2010: 249).

28. Morris (2010: 328).

29. El alcance de este libro, que ha sido traducido a numerosas lenguas, entre elles el castellano, resulta en sí un tema de reflexión, en especial cuando Morris se propone señalar los "Patterns of History and What They Reveal about Future". Es evidente que Morris no conoce o no comparte las teorías de Karl Popper sobre el historicismo y el historismo (vid. Bermejo Barrera, 2009: 277-282); no obstante, sorprende observar a un Catedrático en Arqueología y eminente historiador afirmar ideas de este tipo. on earth, the Persian king ruled under his divine guidance, enabling him to act correctly in moral terms and representing the Good and the Truth (OP arta) against the Evil and the Lie (OP drauga). In this dualism we find the beginnings of the monotheistic religion which later became known as Zoroastrianism, but which at the time of the Achaemenids may more aptly be referred to as Mazdaism. ${ }^{21}$

Más allá de la idea de inmutabilidad atemporal que revela esta confusión, podríamos pensar que una de las razones de ella es, efectivamente, la falta de conocimiento profundo sobre los persas que, hasta hace unas décadas, reinaba impunemente sobre los estudios de la Antigüedad, así como en la profunda dependencia que hasta nuestros mismos días se mantiene sobre las fuentes griegas para el conocimiento de ese otro que para la Hélade representó el mundo persa. ${ }^{22}$ No obstante, es posible que existan otras explicaciones. En cualquier caso, al preguntarnos por el impacto de la visión que hemos señalado como errónea de la religión de los aqueménidas en los historiadores, nos encontramos con ejemplos como los de Gordon Childe, quien en su libro The Aryans. A Study in Indo-European Origins afirmaba sobre el Imperio Persa lo siguiente:

Its official religion, Zoroastrianism, was inspired by genuinely moral conceptions and was international in its appeal and monotheistic in its essence. ${ }^{23}$

En esta apreciación, coincide Gordon Chile con otros personajes de su época, como Gobineau, ${ }^{24}$ el padre de la teoría antisemita convertida posteriormente en dogma intelectual por el nazismo, o Franz Altheim. ${ }^{25}$ Parece que, en este sentido, la influencia sobre los historiadores en relación con la percepción positiva de la antigua Persia (a causa de su religión) debió tener ciertamente lazos con las ideas del arianismo y los estudios sobre el trazado de la línea de progreso de la humanidad en función del protagonismo sobre ello de los indoeuropeos. ${ }^{26}$ De algún modo, la afirmación de Kant con que iniciábamos este trabajo parece haber disfrutado de un larguísimo recorrido. Tan largo que todavía sigue tácitamente entre nosotros, si tenemos que guiarnos por lo que firma alguien tan contemporáneo como Ian Morris:

Like Assyrian kings before them, Persia's rulers believed they were on a mission from God. As they saw it, their family, the Achaemenids, represented the earthly interests of Ahuramazda, the god of light and truth, in his eternal struggle with darkness and evil. ${ }^{27}$

Persia's kings did not, but that was because Iranian Zoroastrianism was evolving along similar lines to Christianity anyways. ${ }^{28}$

Las reminiscencias de estas dos afirmaciones con cuanto hemos expuesto son evidentes, y a la vez las consecuencias de dichas evidencias resultan, en definitiva, temibles. ${ }^{29}$ Apuntaba Heleen Sancissi-Weerdenburg sobre un libro de J. M. Cook, The Persian Empire, publicado en 1983, que pese al paso del tiempo, parece que algunos conceptos y apriorismos sobre la antigua Persia parecen sobrevivir al paso del tiempo, y replicarse de manera inconsciente en el imaginario colectivo de los historiadores de la Antigüedad, quienes a su vez eran responsables de traducirlos y transmitirlos al resto de la población. En cualquier 
caso, la eminente aquemenóloga señalaba que quizás el único modo de superar estos apriorismos sea estudiando los moldes intelectuales y literarios en los que se han ido insertando los datos resultantes de estos apriorismos. ${ }^{30}$ Quisiera pensar que el presente estudio es una contribución en esta misma línea.

30. Sancissi-Weerdenburg (1987: 131). Por otra parte, esta creencia de un parentesco entre las razas indoeuropeas, y por tanto, entre los persas y al menos "algunos" europeos puede advertirse ya en Herder (1959: 367): “los alemanes tenemos motivos de congratularnos de nuestro probable parentesco racial con este pueblo". 


\section{Q Bibliografía}

»Antela-Bernárdez, B. (en prensa). Alejandro ante el nazisme: Franz Altheim, en: Gonzales, A. (ed.), Homenaje a Amparo Pedregal. Besançon, Presses Universitaires de Franche-Comté.

"Antela-Bernárdez, B. y Zaragozà, C. (en prensa). Los persas como esclavos en la historiografía, en: Alvar, A. (ed.), Historiografía de la esclavitud. Getafe, Ediciones de la Universidad Carlos III.

»Bermejo Barrera, X. C. (2009). Introducción a la historia teórica. Madrid, Akal.

»Blok, J. (1994). Quest for the Scientific Mythology: F. Creuzer and K. O. Müller on History and Myth, en: Grafton, A. y Marchand, S. L. (eds.), Proof and Persuasion in History. Middletown (CT), Wesleyan University Press: 26-52.

"Bossuet, J. B. (1996). Discours sur l'histoire universelle. París, Garnier-Flammarion.

"Briant, P. (2005). The Theme of 'Persian Decadence' in Eighteenth-Century European Historiography: Remarks on the Genesis of a Myth, en: Curtis, J. y Simpson, S. J. (eds.), The World of Achaemenid Persia. History, Art and Society in Iran and the Ancient Near East. Londres, I. B. Tauris: 3-16.

»Briant, P. (2009). Alexander and the Persian Empire: Between 'Decline' and 'Renovation', en: Heckel, W. y Tritle, L. A. (eds.), Alexander the Great. A New History. Oxford, Oxford University Press: 171-188.

» Briant, P. (2016). Arthur de Gobineau (1816-1882) entre Darius et Alexandre, en: Binder, C., Börm, H. y Luther, A. (eds.), Diwan. Studies in the History and Culture of the Ancient Near East and the Eastern Mediterranean - Untersuchungen zu Geschichte und Kultur des Nahen Ostens und des östlichen Mittelmeerraumes im Altertum. Festschrift für JosefWiesehöfer zum 65. Geburtstag. Duisburg, Wellem Verlag: 735-756.

» Brosius, M. (2006). The Persians. An Introduction. Londres, Routledge.

»Cartledge, P. (2004). Los griegos. Barcelona, Crítica.

»Creuzer, F. (1825). Religions de l'Antiquité. París, Treuttel et Würtz.

» de Jong, A. (1997). Traditions of the Magi. Zoroastrism in Greek and Latin Literature. Leiden, E. J. Brill.

»Gordon Childe, V. (1926). The Aryans. A Study in Indo-European Origins. Londres, K. Paul, Trench, Trubner \& Co.

» Hegel, G. W. F. (1994). Lecciones sobre la filosofía de la historia universal. Madrid, Alianza.

» Herder, J. G. (1959). Ideas para una filosofía de la historia de la humanidad. Buenos Aires, Losada.

»Imanpour, M. I. (2013). Development of Achaemenid Studies in the West after 1979, en: Analytica Iranica 4: 91-141.

»Imanpour, M. I. (2015). Re-establishment of Achaemenid History and Its Development in the Nineteenth and Twentieth Centuries, en: Iranian Studies 48: 515-530. 
» Kant, I. (1923). Kants gesammelte Schriften. Bd. XV: Anthropologie, Hrsg. von der Preußischen und der Deutschen Akademie der Wissenschaften. Berlín, Deutsche Akademie der Wissenschaften.

»Kreyenbroek, P. G. (2005). Zoroastrism under the Achaemenians: A NonEssential Approach, en: Curtis, J. y Simpson, S. J. (eds.), The World of Achaemenid Persia. History, Art and Society in Iran and the Ancient Near East. Londres, I. B. Tauris: 103-110.

» Malandra, W. W. (1983). An Introduction to Ancient Iranian Religion. Leiden, E. J. Brill.

" Morris, I. (2010). Why the West Rules - For Now. The Patterns of History, and What They Reveal about the Future. Nueva York, Picador.

»Motadel, D. (2013). Iran and the Aryan Myth, en: Ansari, A. M. (ed.), Perceptions of Iran: History, Myths and Nationalism from Medieval Persia to the Islamic Republic. Londres, I. B. Tauris: 119-145.

»Pringle, H. (2006). The Master Plan. Himmler's Scholars and the Holocaust. Nueva York, Hyperion.

"Samiei, S. (2014). Ancient Persia in Western Historiography. Hellenism and the Representation of the Achaemenid Empire. Londres, I. B. Tauris.

"Sancisi-Weenderburg, H. (1987). The Fifth Oriental Monarchy and Hellenocentrism. Cyropedia VIII and Its Influence, en: Sancisi-Weenderburg, H. y Kuhrt, A. (eds.), Achaemenid History II: The Greek Sources. Leiden, E. J. Brill: 117-131.

» Spengler, O. (1926). The Decline of the West. Nueva York, Alfred A. Knopf.

» Toynbee, A. (1972). A Study of History. Londres, Oxford University Press.

»Vázquez Lobeiras, Mํㅗ J. (2008). Oriente y Occidente en la geografía de Kant: con un excurso hacia la antropología y la filosofía de la Historia, en: Vázquez Lobeiras, M. J. y Veiga Rodríguez, A. (eds.), Perspectivas sobre oriente y occidente: actas el II Curso de Primavera, Lugo, 3-7 de abril de 2005. Lugo, Servicio de Publicacions da Universidade de Santiago de Compostela: 209-222.

»Voltaire. (1962). Essai sur les moeurs et l'esprit des nations. París, Sociales.

»Zia-Ebrahimi, R. (2011). Self-Orientalization and Dislocation: The Uses and Abuses of the Aryan Discourse in Iran, en: Iranian Studies 44: 447-452.

»Zia-Ebrahimi, R. (2016). Better a Warm Hug than a Cold Bath: Nationalist Memory and the Failures of Iranian Historiography, en: Iranian Studies 49: 837-854. 
